(c) 2005 International Press

Adv. Theor. Math. Phys. 9 (2005) 315-320

\title{
On Calabi-Yau supermanifolds
}

\author{
Martin Roček and Neal Wadhwa
}

C.N. Yang Institute for Theoretical Physics

SUNY, Stony Brook, NY 11794-3840, USA

rocek@insti.physics.sunysb.edu

\begin{abstract}
We prove that a Kähler supermetric on a supermanifold with one complex fermionic dimension admits a super Ricci-flat supermetric, if and only if the bosonic metric has vanishing scalar curvature. As a corollary, it follows that Yau's theorem does not hold for supermanifolds.
\end{abstract}

Calabi [1] proposed that if a Kähler manifold has vanishing first Chern class, that is, the Ricci-form obeys $R_{i \bar{j}}(g)=\partial_{i} \bar{v}_{j}-\bar{\partial}_{j} v_{i}$ for a globally defined 1-form $v$, or, equivalently, a complex $n$-dimensional Kähler manifold has a globally defined holomorphic top form $\Omega_{i_{1}, \ldots, i_{n}}$, then there exists a unique metric $g^{\prime}$ which is a smooth deformation of $g$ and obeys $R_{i \bar{j}}\left(g^{\prime}\right)=0$. Yau $[\mathbf{2}]$ proved this theorem for ordinary manifolds.

Recently, there has been a lot of interest in Calabi-Yau supermanifolds [3-5]; though these papers use only the topological properties of such spaces, it is interesting to ask whether they also admit Ricci-flat supermetrics. This paper studies the generalization of Calabi's conjecture to supermanifolds with one complex fermionic dimension. We find that such a Kähler supermanifold admits a Ricci-flat supermetric if and only if the bosonic metric has vanishing scalar curvature. For a given scalar-flat bosonic Kähler metric with Kähler potential $K_{\text {Bose }}$, the super-extension is unique, and has the

e-print archive: http://lanl.arXiv.org/abs/hep-th/0408188 
super Kähler potential:

$$
K\left(z^{i}, \bar{z}^{j}, \theta, \bar{\theta}\right)=K_{\text {Bose }}\left(z^{i}, \bar{z}^{j}\right)+\operatorname{det}\left(\frac{\partial^{2}}{\partial z^{i} \partial \bar{z}^{j}} K_{\text {Bose }}\right) \theta \bar{\theta} .
$$

As complex projective spaces do not admit scalar-flat metrics, but do admit super Calabi-Yau extensions with one fermionic dimension, it follows that Yau's theorem does not hold for supermanifolds.

A supermanifold is a generalization of a usual manifold with fermionic as well as bosonic coordinates. ${ }^{1}$ The bosonic coordinates are ordinary numbers, whereas the fermionic coordinates are grassmann numbers. Grassmann numbers are odd elements of a grassmann algebra and anticommute: $\theta^{1} \theta^{2}=-\theta^{2} \theta^{1}$ and $\theta^{1} \theta^{1}=0$.

On bosonic Kähler manifolds, the Ricci tensor

$$
R_{i \bar{j}}=-(\ln \operatorname{det}(g))_{, i \bar{j}}
$$

For this to vanish, ln $\operatorname{det}(g)$ ) (locally) must be the real part of a holomorphic function, and hence, $\operatorname{det}(g))=|f(z)|^{2}$ for some holomorphic $f(z)$. This can always be absorbed by a holomorphic coordinate transformation, and hence a Kähler manifold is Ricci-flat if its Kähler potential $K$ obeys the MongeAmpère equation

$$
\operatorname{det}(g) \equiv \operatorname{det}\left(K_{, i \bar{j}}\right)=1 .
$$

On supermanifolds, because elements of $g$ contain grassmann numbers, the determinant is not well-defined and a new definition of the determinant is needed. For any non-degenerate supermatrix

$$
g=\left(\begin{array}{ll}
A & B \\
C & D
\end{array}\right)
$$

where $A$ and $D$ are bosonic and $B$ and $C$ are fermionic,

$$
\operatorname{sdet}(g) \equiv \frac{\operatorname{det}(A)}{\operatorname{det}\left(D-C A^{-1} B\right)}=\frac{\operatorname{det}\left(A-B D^{-1} C\right)}{\operatorname{det}(D)} .
$$

For arbitrary supermatrices $X, Y$, this definition is consistent with the basic relation $\operatorname{sdet}(X Y)=\operatorname{sdet}(X) \operatorname{sdet}(Y)$. In addition, the supertrace is defined as

$$
\operatorname{str}(g)=\operatorname{tr}(A)-\operatorname{tr}(D)
$$

\footnotetext{
${ }^{1}$ More rigorous and technical definitions can be found in the literature (see e.g., $[\mathbf{6}, \mathbf{7}]$, but this simple treatment suffices for our results.
} 
which is consistent with $\operatorname{str}(X Y)=\operatorname{str}(Y X)$. These two definitions imply an identity that is useful in simplifying expressions that use grassmann numbers:

$$
\ln \operatorname{sdet}(g)=\operatorname{str} \ln (g)
$$

Simple examples [5] of Kähler supermanifolds are provided by superprojective spaces, $S P(m \mid n)$. These can be described in terms of $m+n+1$ homogeneous coordinates:

$$
\left(z^{1}, z^{2}, \ldots, z^{m+1} \mid \theta^{1}, \ldots, \theta^{n}\right)
$$

related by the equivalence relations $z^{i} \sim \lambda z^{i}$ and $\theta^{i} \sim \lambda \theta^{i}$. There are $m+1$ coordinate patches where $z^{i} \neq 0$ in the $i$-th coordinate patch. In the $i$-th patch, we can introduce inhomogeneous coordinates $\tilde{z}^{j}=z^{j} / z^{i}$. Other examples include weighted superprojective space, $W S P\left(k_{1}, \ldots, k_{m+1}\right.$ $\left.\mid l_{1}, \ldots, l_{n}\right)$; the coordinates are identified under the equivalence relations $z^{i} \sim \lambda^{k_{i}} z^{i}$ and $\theta^{i} \sim \lambda^{l_{i}} \theta^{i}$. A direct calculation of the Ricci-form of the standard Fubini-Study metric reveals that $S P(m \mid m+1)$ are Calabi-Yau and have a vanishing Ricci-form, whereas $W S P(1, \ldots, 1 \mid m)$ are Calabi-Yau but have a non-vanishing Ricci-form (see below).

We now show that for an arbitrary Kähler space with only one complex fermionic coordinate, $R_{i \bar{j}}=0$ implies that the bosonic part of the Kähler potential yields a space with a Ricci scalar $s=0$. Consider an arbitrary super Kähler potential $K$, on a supermanifold $\mathcal{M}(m \mid 1)$ with one complex fermionic coordinate $\theta$ and $m$ bosonic coordinates. The super Kähler potential can be written as $K=f^{0}+f^{1} \theta \bar{\theta}$. We use the convention that holomorphic derivatives are taken from the left and anti-holomorphic derviatives are taken from the right. The supermetric $\mathbf{g}$ is the block matrix

$$
\mathbf{g}=\left(\begin{array}{cc}
f_{, i \bar{j}}^{0}+f_{, i \bar{j}}^{1} \theta \bar{\theta} & f_{, i}^{1} \theta \\
f_{, \bar{j}}^{1} \bar{\theta} & f^{1}
\end{array}\right) .
$$

Its superdeterminant is

$$
\begin{aligned}
\operatorname{sdet}(\mathbf{g}) & =\frac{\operatorname{det}\left[f_{, i \bar{j}}^{0}+\left(f_{, i \bar{j}}^{1}-f_{, i}^{1} f_{, \bar{j}}^{1} / f^{1}\right) \theta \bar{\theta}\right]}{f^{1}} \\
& =\frac{\operatorname{det}\left(f_{, i \bar{j}}^{0}\right)}{f^{1}} \operatorname{det}\left[\delta_{i}^{k}+g^{k \bar{j}}\left(f_{, i \bar{j}}^{1}-\frac{f_{, i}^{1} f_{, \bar{j}}^{1}}{f^{1}}\right) \theta \bar{\theta}\right],
\end{aligned}
$$

where $g^{i \bar{j}} \equiv\left(f_{, i \bar{j}}^{0}\right)^{-1}$ is the inverse metric of the bosonic manifold. Using the identity (1.7), we can rewrite this as:

$$
\operatorname{sdet}(\mathbf{g})=\frac{\operatorname{det}\left(f_{, i \bar{j}}^{0}\right)}{f^{1}}\left[1+g^{i \bar{j}}\left(f_{, i \bar{j}}^{1}-\frac{f_{, i}^{1} f_{, \bar{j}}^{1}}{f^{1}}\right) \theta \bar{\theta}\right] .
$$


On a super Ricci-flat manifold, the superdeterminant can be chosen to be 1. The $\theta$-independent term of $\operatorname{sdet}(\mathbf{g})=1$ implies

$$
f^{1}=\operatorname{det}\left(f_{, i \bar{j}}^{0}\right) \text {. }
$$

The remaining term must vanish on a super Ricci-flat Kähler manifold. This implies

$$
g^{i \bar{j}}\left(f_{, i \bar{j}}^{1}-\frac{f_{, i}^{1} f_{, \bar{j}}^{1}}{f^{1}}\right)=f^{1} g^{i \bar{j}}\left[\ln \left(f^{1}\right)\right]_{, i \bar{j}}=0 .
$$

Substituting (1.12) implies

$$
g^{i \bar{j}} \ln \operatorname{det}\left(f_{, l \bar{k}}^{0}\right)_{, i \bar{j}} \equiv g^{i \bar{j}} R_{i \bar{j}}=0,
$$

which is precisely the Ricci scalar of the bosonic space with Kähler potential $f^{0}$. This proves our main result: a Kähler supermanifold with one complex fermionic dimension admits a super Ricci-flat extension, if and only if the bosonic Kähler manifold that it is based on has vanishing scalar curvature $s$. Many such bosonic manifolds are known and have been studied; (see e.g., [9-11]; such spaces all admit supermanifolds with Calabi-Yau supermetrics. A simple example is the space $\mathbb{C P}^{1} \times \Sigma$, where $\Sigma$ is a Riemann surface with a metric with constant curvature chosen so that the total scalar curvature vanishes. The super Ricci-flat Kähler potential on such a space is

$$
K=\ln \left(1+z_{1} \bar{z}_{1}\right)-\ln \left(1-z_{2} \bar{z}_{2}\right)+\frac{\theta \bar{\theta}}{\left(1+z_{1} \bar{z}_{1}\right)^{2}\left(1-z_{2} \bar{z}_{2}\right)^{2}} .
$$

There are many other $s=0$ metrics which can be studied this way.

A corollary of our result is that there are many Kähler supermanifolds with vanishing first Chern class that do not admit super Ricci-flat supermetrics, thus proving that Yau's theorem does not apply to supermanifolds. Clearly, since no projective space admits an $s=0$ metric, no supermanifold with one complex fermionic coordinate that is based on projective space admits a super Ricci-flat supermetric. To find our counterexample, it suffices to prove that such supermanifolds may have vanishing first Chern class.

We now consider the explicit example $W S P(1,1 \mid 2)$. The superprojective space $W S P(1,1 \mid 2)$ has a bosonic base which is just $\mathbb{C P}^{1}$, and $\ln \operatorname{det}(\mathbf{g})$ of the Fubini-Study supermetric is the globally defined scalar, $\theta \bar{\theta} /(1+z \bar{z})^{2}$. The gradient of this scalar is a globally defined vector that fulfills the conditions of the super Calabi-Yau conjecture. Equivalently, the top form $d z \wedge d \theta$ is a globally defined holomorphic top-form (the superdeterminant of the coordinate transformation $z \rightarrow-1 / z, \theta \rightarrow \theta / z^{2}$ between the two patches that 
cover $\mathbb{C P}^{1}$ is 1$)$. As the bosonic part of $W S P(1,1 \mid 2), \mathbb{C P}^{1}$, has no metric with Ricci scalar $s=0$, this space does not satisfy the super Calabi-Yau conjecture. This result can be generalized to $W S P(\underbrace{1, \ldots, 1}_{m} \mid m)$. These spaces have a globally defined vector on them that fulfill the conditions of the super Calabi-Yau conjecture or equivlently they have globally defined holomorphic top-forms that exist in every coordinate patch. In [8], it is observed that $\operatorname{WSP}(1,1 \mid 2)$ appears to violate the super Calabi-Yau conjecture, though no explicit proof is given, and it is conjectured that $W S P$ $(1, \ldots, 1 \mid m)$ for $m>2$ will satisfy the conjecture; here we have shown that no $W S P(1, \ldots, 1 \mid m)$ admits a super Ricci-flat supermetric.

\section{Acknowledgment}

We are happy to thank C. LeBrun, S. Sethi, C. Vafa, E. Witten, and S.T. Yau for encouragement and helpful comments, and the Second Simons Workshop in Physics and Mathematics for a stimulating environment. The work of MR was supported in part by NSF grant no. PHY-0354776.

\section{References}

[1] E. Calabi, The space of Kahler metrics Proc. Int. Congr. Math. Amsterdam 2, 206-207.

[2] S.-T. Yau, On the Ricci curvature of a compact Kahler manifold and the Monge-Ampere equations. I, Commun. Pure Appl. Math. 31 (1978), 339 .

[3] E. Witten, Perturbative gauge theory as a string theory in twistor space, arXiv:hep-th/0312171.

[4] A. Neitzke and C. Vafa, $N=2$ strings and the twistorial Calabi-Yau, arXiv:hep-th/0402128.

[5] M. Aganagic and C. Vafa, Mirror symmetry and supermanifolds, arXiv:hep-th/0403192.

[6] B. DeWitt, Supermanifolds Cambridge. Second edition. University Press of Cambridge, UK Monographs on mathematical physics, 1992.

[7] D.S. Freed, IAS/Park City Lectures on Classical Field Theory and Supersymmetry IAS/Park City Mathematics series 11 (2001), http://www.ma.utexas.edu/users/dafr/pcmi.pdf 
[8] S. Sethi, Supermanifolds, rigid manifolds and mirror symmetry, Nucl. Phys. B 430 (1994), 31; arXiv:hep-th/9404186.

[9] C. LeBrun, Scalar-flat Kaehler metrics on blown-up ruled surfaces, J. reine Angew. Math. 420 (1991), 161-177.

[10] C. LeBrun, On the scalar curvature of complex surfaces, Geom. Func. Anal. 5 (1995), 619-628.

[11] C. LeBrun, J.-S. Kim and M. Pontecorvo, Scalar-flat Kaehler surfaces of all genera, J. reine Angew. Math. 486 (1997), 69-95. 